\title{
Depth image based on rehabilitation human behavior recognition (Withdrawal Notice)
}

Xiaohong Tong, Shanshan Ma, Chao Tang, Li Li, Dandan Liang, et al.

Xiaohong Tong, Shanshan Ma, Chao Tang, Li Li, Dandan Liang, Rongjun Wang, Ling Chen, Congying Song, "Depth image based on rehabilitation human behavior recognition (Withdrawal Notice)," Proc. SPIE 11565, AOPC 2020: Display Technology; Photonic MEMS, THz MEMS, and Metamaterials; and $\mathrm{Al}$ in Optics and Photonics, 115650K (5 November 2020); doi: $10.1117 / 12.2576129$

SPIE Event: Applied Optics and Photonics China (AOPC 2020), 2020, Beijing, China 


\section{Depth image based on rehabilitation human behavior recognition (Withdrawal Notice)}

Xiaohong Tong and Shanshan Ma

Anhui Agricultural Univ. (China)

Chao Tang

Hefei Univ. (China)

Lا Li, Dandan Liang, Rongjun Wang, Ling Chen, and Congying Song

Hefei Technology College (China)

Proceedings Volume 11565, AOPC 2020: Display Technology; Photonic MEMS, THz MEMS, and

Metamaterials; and Al in Optic sand Photonic s; 115650K (2020) https://doi.org/10.1117/12.2576129

Event Applied Optics and Photonics China (AOPC2020), 2020, Beijing, China

Online Publication Date: 5 November 2020

Withdrawn from Publication: 13 November 2020

Publisher's Note: This paper, origina lly published 5 November 2020, wa s withdra wn per author request. 